\title{
Pneumocystis jirovecii dihydropteroate synthase gene mutations in a group of HIV-negative immunocompromised patients with Pneumocystis pneumonia
}

\author{
YINGJIAO LONG, CHENG ZHANG, LI SU and CHENGLI QUE \\ Department of Pulmonary Medicine, First Hospital, Peking University, Beijing 100034, P.R. China
}

Received March 5, 2014; Accepted September 4, 2014

DOI: $10.3892 /$ etm.2014.2002

\begin{abstract}
The purpose of this study was to investigate dihydropteroate synthase (DHPS) mutations and their clinical context in non-HIV-infected patients with Pneumocystis pneumonia (PCP). DHPS genes in respiratory samples collected from HIV-negative patients with PCP presented between January 2008 and April 2011 were amplified by polymerase chain reaction (PCR) and sequenced. Basic clinical data from the medical records of the patients were also reviewed. The most common point mutations, which result in Thr55Ala and Pro57Ser amino acid substitutions, were not detected in the Pneumocystis jirovecii sampled from the HIV-negative patients. Two other point mutations, which result in nonsynonymous mutation, Asp90Asn and Glu98Lys, were identified in P.jirovecii from two patients. Among the patients, the levels of lactate dehydrogenase ( $\mathrm{LDH}), \mathrm{C}$-reactive protein (CRP) and plasma (1-3) $\beta$-D-glucan were elevated in 75, 92.31 and $42.86 \%$ of patients, respectively. The percentage of circulating lymphocytes was significantly lower in non-survivors than in survivors [4.2\%, interquartile range (IQR) 2.4-5.85 versus $10.1 \%$, IQR 5.65-23.4; $\mathrm{P}=0.019]$. The neutrophil proportion in bronchoalveolar lavage fluid (BALF) was significantly higher in non-survivors than in survivors $(49.78 \pm 27.67$ versus $21.33 \pm 15.03 \% ; \mathrm{P}=0.047)$. Thirteen patients had received adjunctive corticosteroids ( $1 \mathrm{mg} / \mathrm{kg} /$ day prednisone equivalent) and nine $(69.23 \%)$ of them eventually experienced treatment failure. No common DHPS gene mutations of $P$.jirovecii were detected in the HIV-negative PCP patients. However, other mutations did exist, the significance of which remains to be further identified. The elevation of neutrophil counts in BALF and reduction of the number of lymphocytes in peripheral blood may be associated with poor outcome. The efficacy
\end{abstract}

Correspondence to: Dr Chengli Que, Department of Pulmonary Medicine, First Hospital, Peking University, 8 Xishiku Street, Beijing 100034, P.R. China

E-mail: quechengli@hotmail.com

Key words: Pneumocystis jirovecii, drug resistance, dihydropteroate synthase gene, mutation of adjunctive steroid therapy in HIV-negative patients with $P$.jirovecii infection requires further investigation.

\section{Introduction}

Pneumocystis pneumonia (PCP), which is caused by Pneumocystis jirovecii (formerly called Pneumocystis carinii f. sp.hominis), is among the most prevalent opportunistic infections among immunocompromised patients, especially in patients with AIDS. In the past, PCP was generally indolent with latent onset; however, in HIV-negative patients, it usually progresses rapidly to severe hypoxemia, or even respiratory failure (1) with high mortality.

Currently, co-trimoxazole, (a 1:5 mixture of trimethoprim and sulfamethoxazole; TMP-SMZ) remains the first-line and most effective regimen for anti-Pneumocystis therapy and has been widely used for prophylaxis in patients at high risk for PCP since the 1990s. The widespread application of co-trimoxazole has been implicated in the increases in sulfa drug-resistant bacteria reported in HIV-infected patients. It has also raised concerns about the possible selection of drug-resistant Pneumocystis. In addition, a number of studies have confirmed that mutations in the Pneumocystis dihydropteroate synthase (DHPS) gene, which encodes the target enzyme that is combined with and inhibited by sulfamethoxazole, confer the sulfa drug resistance. The most frequently reported mutations are at positions corresponding to codons 55 and 57, which result in amino acid changes $(2,3)$, that is, nonsynonymous mutation. Furthermore, there are also reports of mutations on codons 23 (2), 111 (2), 248 (2), 171 (4) and 60 (5).

So far, genetic and epidemiological data concerning $P$. jirovecii infections in China are rather scarce, and mainly relate to isolates from $\mathrm{HIV}$-infected patients. Kazanjian et al (6) revealed a DHPS gene mutation rate of $7 \%$ (1/15) in AIDS patients with PCP in Beijing. However, Li et al (7) did not find mutations in the DHPS gene in P. jirovecii in $10 \mathrm{HIV}$-infected patients in Guangzhou, China. To the best of our knowledge, no data have been reported concerning Pneumocystis DHPS gene mutations in HIV-negative patients in China.

The objective of the present study was to investigate the presence of DHPS gene mutations in $P$. jirovecii from non-HIV-positive patients with PCP from a major general hospital in China. Clinical information, including certain laboratory parameters, treatment and outcome 
were also reviewed, to determine the effect of mutation of the Pneumocystis DHPS gene on clinical outcome in HIV-negative patients.

\section{Methods}

Patients. In this retrospective study, 22 non-HIV-positive patients with PCP, which were confirmed by Gomori methenamine silver (GMS) staining of respiratory samples, were included. The patients attended Peking University First Hospital, a 1,368-bed teaching hospital in Beijing, China between January 2008 and April 2011. The present study was approved by the Institutional Review Board of Peking University and was performed in accordance with the recommendations of the Helsinki Declaration of 1975. Written informed consent was obtained from all patients.

Materials. A total of 24 respiratory samples, comprising 22 bronchoalveolar lavage fluid (BALF) and two sputum samples, were obtained from the 22 HIV-negative patients with confirmed PCP. The patients' medical records were also reviewed and the outcome was followed up.

Sample processing and DNA extraction. All the samples were dissolved with dithiothreitol (DTT) first, and then filtered with a nylon mesh and centrifugation. Part of each sample was stained and demonstrated to be GMS positive, and the remainder was stored at $-20^{\circ} \mathrm{C}$. DNA extraction was performed using E.Z.N.A. Blood DNA kit (Omega Bio-Tek Inc., Norcross, GA, USA).

Polymerase chain reaction (PCR). PCR was performed to analyze the DHPS gene of Pneumocystis, using the previously reported primers DHPS-3 and DHPS-4 (8). The reaction mixture contained $2.5 \mu \mathrm{l}$ DNA template, $12.5 \mu \mathrm{l}$ Taq PCR Master Mix (Qiagen, Valencia, CA, USA), $1.5 \mu 1$ $(10 \mu \mathrm{M})$ forward primer, $1.5 \mu \mathrm{l}(10 \mu \mathrm{M})$ reverse primer and sterile water, making a total volume of $25 \mu \mathrm{l}$. PCR was used to amplify the samples, yielding a 370-bp fragment. A hot-start step at $94^{\circ} \mathrm{C}$ for $5 \mathrm{~min}$ was followed by the following for 45 cycles: DNA denaturation at $94^{\circ} \mathrm{C}$ for $30 \mathrm{sec}$, annealing at $61^{\circ} \mathrm{C}$ for $30 \mathrm{sec}$ and extension at $72^{\circ} \mathrm{C}$ for $30 \mathrm{sec}$. This was followed by a final extension step at $72^{\circ} \mathrm{C}$ for $5 \mathrm{~min}$. The amplification products were analyzed by electrophoresis on a $1.5 \%$ agarose gel containing ethidium bromide, and the bands were visualized with ultraviolet light. To prevent contamination, all PCR procedures were performed with a negative control of sterile water.

Sequencing. The PCR products were sent to a genomic company (SinoGenoMax Co., Ltd, Beijing, China) and sequenced using an automated DNA sequencer (ABI 3730xl; Applied Biosystems, Foster City, CA, USA). Sequence analysis was performed using BLAST (http://blast.ncbi.nlm.nih.gov/Blast. cgi) and Clustal W (http://www.ebi.ac.uk/Tools/msa/) software.

Statistical analysis. Clinical information, including laboratory results, therapy and outcome of patients were collected from medical records. SPSS software, version 17.0 (SPSS,
Table I. Patient demographics at diagnosis of Pneumocystis pneumonia in 18 patients.

\begin{tabular}{ll}
\hline Characteristic & Number \\
\hline Age, years (range) & $51(23-77)$ \\
Male gender (\%) & $12(67)$ \\
Underlying disease (\%) & \\
Inflammatory disease & \\
Pemphigus erythematosus & $1(6)$ \\
ANCA-associated systemic vasculitis & $3(17)$ \\
Connective tissue disease & $3(17)$ \\
Chronic glomerulonephritis & $1(6)$ \\
Malignancy & \\
Solid tumor & $3(17)$ \\
Hematologic malignancy & $2(11)$ \\
Organ transplantation & \\
Bone marrow & $1(6)$ \\
Kidney & $3(17)$ \\
Other & $2(11)$
\end{tabular}

Some patients had two or more underlying conditions. ANCA, ant-neutrophil cytoplasmic antibody.

Inc., Chicago, IL, USA) was used to analyze data, and the Student's t-test was used to assess significant differences in continuous data with Gaussian distribution, while the Mann-Whitney U-test was used for non-normal distribution. Proportions between groups were compared using the $\chi^{2}$ test. A P-value of $<0.05$ was considered to indicate a statistically significant result.

\section{Results}

Demographic and clinical characteristics. Twenty-two specimens from bronchoalveolar lavage and two sputum samples were obtained from 22 HIV-negative patients with confirmed PCP from January 2008 to April 2011. Twenty-one DHPS gene fragments (that include the most frequently reported mutations) from 20 patients were successfully extracted from $24(87.5 \%)$ samples. Among these 20 patients, two patients were outpatients whose medical records were unable to be retrieved. The demographic characteristics of the other 18 patients and underlying diseases of these patients are listed in Table I. All patients had received immunosuppressive agents, but none of them had ever received prophylaxis against PCP. One patient (5.6\%) had experienced a prior episode of PCP. Table II shows that lactate dehydrogenase (LDH) levels were above the upper limit of normal in 9/12 patients (75\%). $\beta$-D-glucan levels were elevated in $10 / 14$ patients $(71.3 \%)$. The $\mathrm{CD}^{+}$lymphocyte count was $<200 / \mu 1$ in $9 / 10$ patients $(90 \%)$.

The 28 -day mortality rate was $50 \%$. Table III shows the prognostic factors that were identified to be associated with mortality by univariate analysis. These were peripheral neutrophils $(\mathrm{P}=0.003)$, peripheral lymphocytes $(\mathrm{P}=0.019)$ and 
Table II. Laboratory parameters in patients with Pneumocystis pneumonia.

Parameter

Value

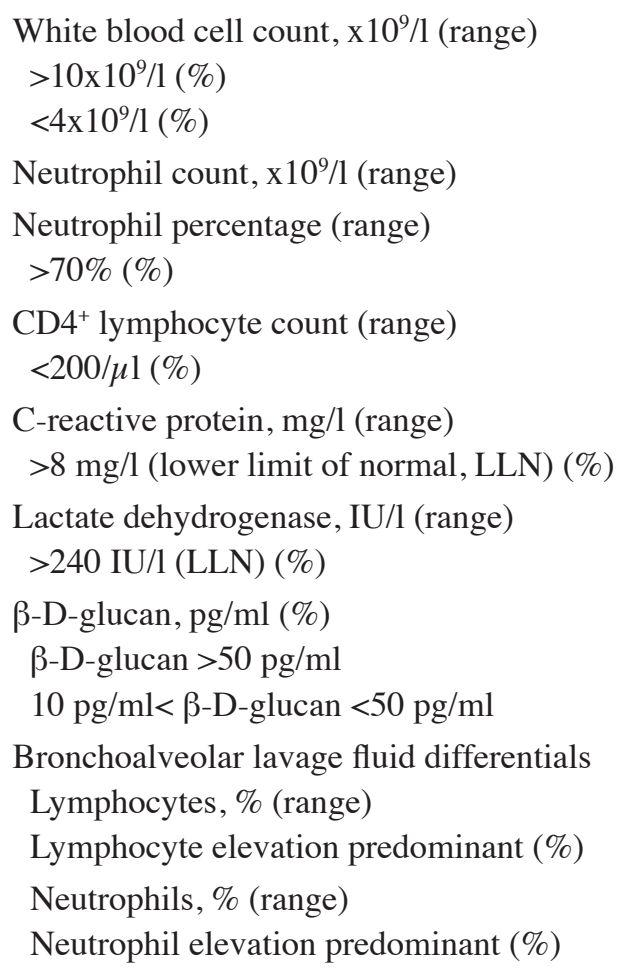

${ }^{a}$ Incomplete data. Data are presented as mean \pm standard deviation, or median and interquartile range.

neutrophils in BALF $(\mathrm{P}=0.047)$. The treatment and related mortality were also analyzed and are summarized in Table IV. Caspofungin therapy was administered to eight patients $(50 \%)$ and $75 \%$ of them failed to survive. Thirteen patients had received adjunctive corticosteroids $(1 \mathrm{mg} / \mathrm{kg} /$ day prednisone equivalent) and nine (69.23\%) of them succumbed.

DHPS gene mutations. No mutations in the DHPS gene were detected in the sequenced amplicons at codons 55 or 57. All had the wild-type genotype with the nucleotide sequence ACA CGG CCT at codons 55, 56 and 57, respectively, corresponding to threonine and proline at positions 55 and 57.

However, gene mutations at two relatively rare positions were identified. One mutation was observed at DHPS codon 98 in two patients with PCP, with glutamate replaced by lysine. The other was at DHPS codon 90 in a sample obtained from one of these two patients, with aspartate replaced by asparagine. The patient with only one mutation at codon 98 , a 51-year-old female with dermatomyositis, developed PCP during adjustment of the dose of corticosteroids and finally succumbed following active treatment with TMP-SMZ. The patient with two mutations was a 31 -year-old male kidney transplant recipient. The manifestations of this patient were mild with a $\mathrm{PaO}_{2}$ of $83.29 \mathrm{mmHg}$ on ambient air. The mycophenolate mofetil dosage of the patient was halved immediately following the diagnosis of PCP, while the FK506 dosage remained unchanged. This patient declined TMP-SMZ treatment and self-discharged. The patient was followed up by telephone and it was reported that his condition had improved.

\section{Discussion}

The present study is, to the best of our knowledge, the first that has investigated DHPS gene mutations in HIV-negative PCP patients in China. The results of the present study revealed that the most common point mutations, which result in Thr55Ala and Pro57Ser amino acid substitutions, were not detected in the $P$. jirovecii isolates from any of the samples from 20 non-HIV patients with confirmed PCP. This result is consistent with that of previous studies in HIV-positive patients $(6,7)$, and suggests that the prevalence of DHPS gene mutations remains low in China. A number of studies have found that the prevalence of DHPS gene mutations in developed countries is relatively higher than that in developing countries with the highest prevalence being $72 \%$ in the USA $(6,9)$. It is hypothesized that the relatively lower prevalence in developing countries, for example, $6.2 \%$ in India (10) and 56\% in Africa (11), may be due to the reduced use of sulfa prophylaxis. It has been reported that even short-term exposure to TMP-SMZ can be associated with the emergence of resistance (12). According to the literature, the major antipneumocystic activity of this agent for PCP is derived from sulfamethoxazole (13) and the trimethoprim component is a very poor inhibitor of $P$.jirovecii DHFR (dihydrofolate reductase) (14). A number of studies have documented an association between the failure of sulfa 
Table III. Prognostic factors.

\begin{tabular}{|c|c|c|c|}
\hline Variable & Non-survivors & Survivors & P-value \\
\hline Age, years & $52.89 \pm 10.89$ & $49.22 \pm 5.18$ & 0.479 \\
\hline Male, no. (\%) & $5(55.6)$ & $7(77.8)$ & 0.331 \\
\hline Peripheral neutrophils, $\%$ & $91.24 \pm 5.18$ & $75.44 \pm 17.18$ & $0.003^{\mathrm{a}}$ \\
\hline Peripheral lymphocytes, $\%$ (range) & $4.2(2.4-5.85)$ & $10.1(5.65-23.4)$ & $0.019^{\mathrm{a}}$ \\
\hline Lactate dehydrogenase, IU/1 & $416 \pm 84.29$ & $435 \pm 355.81$ & 0.908 \\
\hline $\mathrm{CD}^{+}$lymphocyte, $\mathrm{n} / \mu 1$ (range) & $93(54-209)$ & $97(39-165.80)$ & 0.703 \\
\hline C-reactive protein, mg/l (range) & $69.1(13.5-140)$ & $55.15(26.1-89.55)$ & 0.454 \\
\hline$\beta$-D-glucan >50 pg/ml (\%) & $4 / 6(66.6)$ & $2 / 8(25.0)$ & 0.170 \\
\hline$\beta$-D-glucan >10 pg/ml (\%) & $5 / 6(83.3)$ & $5 / 8(62.5)$ & 0.580 \\
\hline $\mathrm{PaO}_{2} / \mathrm{FiO}_{2}$ & $164.79 \pm 78.44$ & $245.73 \pm 114.32$ & 0.106 \\
\hline Lymphocytes in BALF, \% & $18.44 \pm 18.60$ & $31.00 \pm 11.80$ & 0.107 \\
\hline Neutrophils in BALF, \% & $49.78 \pm 27.67$ & $21.33 \pm 15.03$ & $0.047^{\mathrm{a}}$ \\
\hline
\end{tabular}

${ }^{a}$ Multivariate analysis cannot be achieved due to small sample size. BALF, bronchoalveolar lavage fluid; $\mathrm{PaO}_{2}$, partial pressure of oxygen in arterial blood; $\mathrm{FiO}_{2}$, fraction of inspired oxygen.

Table IV. Pneumocystis pneumonia treatment and outcome.

\begin{tabular}{lcccc}
\hline Treatment & Total & Non-survivors & Survivors & Mortality $(\%)$ \\
\hline TMP-SMZ & 16 & 8 & 8 & 50 \\
TMP-SMZ + caspofungin & 8 & 6 & 2 & 75 \\
Primaquine + clindamycin & 3 & 2 & 1 & 66.67 \\
Adjunctive corticosteroids & 13 & 9 & 4 & 69.23 \\
\hline
\end{tabular}

TPM, trimethoprim; SMZ, sulfamethoxazole.

prophylaxis and the occurrence of mutations in the $P$.jirovecii gene coding for DHPS, especially at codons 55 and 57, either as single mutations or combined $(15,16)$. One study reported that DHPS mutations were significantly more common in patients who had previous exposure to sulfa drugs $(18 / 29 ; 62 \%)$ than in those who had no exposure $(13 / 123,10.5 \%$; $\mathrm{P}<0 \cdot 0001)(5)$. In another study, which included 158 patients of five hospitals in France, multivariate analysis of risk factors for DHPS gene mutation revealed that sulfa prophylaxis is among the independent risk factors (adjusted odds ratio, 26.04; $\mathrm{P}<0.001$ ) (5).

It has been suggested that the geographic area of residence, which may reflect the resistant strains, is an independent predictor of the harboring of DHPS mutations (17). No common mutations of the DHPS gene were detected in the present study. This result may be associated with the characteristics of the population that was studied. Kazanjian et al (18) have also reported that the duration of prophylaxis increases the risk of mutations [relative risk ( RR) for each exposure month, 1.06; $\mathrm{P}=0.02$ ] and that there is a statistically significant increase in the presence of a DHPS mutation if the duration of sulfa exposure is $>1$ year $(\mathrm{RR}, 1.16 ; \mathrm{P}=0.003)$; however, the dose of sulfa was not found to be significantly associated with the mutation. The present study focused on HIV-negative patients and none of them received any sulfa prophylaxis. Although some of the patients in the present study (10/18) received sulfa drug treatment prior to BALF sampling, this was of little consequence due to the short exposure time ( $<7$ days).

Patients with mutant DHPS genotypes are more likely to have severe disease, require invasive ventilation and have a poor outcome than patients with wild-type DHPS genotypes $(19,20)$. In the current study, $72.2 \%$ of patients had hypoxemia and $66.7 \%$ of patients received mechanical ventilation. Although TMP-SMZ was used as first-line treatment, with the exception of contraindication as soon as PCP was suspected, the 28-day mortality rate remained as high as $50 \%$. It is assumed that DHPS gene mutation may be just one of the mechanisms of sulfa resistance. A number of other factors may also play a role, such as pharmacokinetics/pharmacodynamics (PK/PD), underlying diseases, nutritional status and complications. The timing of the first dose of sulfa administration is likely to be crucial to the outcome of patients. In addition, the full length of the DHPS gene was not examined to exclude the possibility of existence that other mutation positions exist that are responsible for sulfa-drug resistance, because a previous study has done so and found no such mutations (5). As certain patients harbouring Pneumocystis with DHPS gene mutations respond to treatment with high doses of TMP-SMX, a 
possible explanation is that high-dose therapy may compensate for reduced sensitivity (21). However, in the present study, all patients received $15 \mathrm{mg} / \mathrm{kg}$ of TMP, or an adjusted dose according to renal function.

With the absence of common mutations, two other nonsynonymous point mutations, Asp90Asn and Glu98Lys, were identified in the $P$. jirovecii isolates from two patients who had different underlying diseases and clinical manifestations, as well as completely different outcomes. The implications of these mutations call for larger scale study.

The immune backgrounds of the patients in the current study were similar to those in a previous study (9). However, none of the patients in the present study had ever received prophylaxis, despite the fact that in nine of 10 patients, the $\mathrm{CD}^{+}$lymphocyte count was $<200 / \mu 1$ on admission. It has been suggested that prophylactic treatment should be applied to HIV-positive patients with $\mathrm{CD}^{+}$lymphocyte counts $<200 / \mu 1$ (10). However, there is no such agreement for HIV-negative immunosuppressed patients. In clinical practices, physicians have to weigh benefits against risks to make an appropriate decision.

The onset symptoms of PCP were variable and nonspecific, including fever, cough, dyspnea and chest tightness. It has been suggested that the level of serum $\beta$-D-glucan and $S$-adenosylmethionine is diagnostic for PCP within the appropriate clinical context (22) and the level of LDH is elevated at an early stage, offering diagnostic value despite its low specificity (23). In the present study, $75 \%$ of patients had elevated LDH levels and $92.31 \%$ of them had elevated CRP levels, supporting the high sensitivity of these indicators. In addition, $71.43 \%$ of patients had elevated $\beta$-D-glucan levels, which is lower than the sensitivity of $98 \%$ and specificity of $94 \%$ reported by a previous study (24). The results of the present study showed that serum $\beta$-D-glucan levels were markedly elevated in $42.86 \%$ of patients; however, the reduction of the level with therapy did not translate into survival. At present, the gold standard to confirm PCP remains the microscopic examination of respiratory samples. However a low fungal load in HIV-negative patients with PCP (25) may lead to false negativity (26). Several PCR assays have been developed with higher sensitivity and specificity, but the detection of Pneumocystis DNA provides no information concerning the organism's viability or infectivity, and therefore cannot exclude the possibility of colonization in asymptomatic patients (27), particularly in patients receiving corticosteroid therapy or immunocompetent patients with lung disease $(28,29)$. In the present study, $55.6 \%$ of the patients received sulfa pre-emptive treatment prior to bronchoalveolar lavage, which may have exerted an influence on the positive rate of microscopy. The results showed that the lymphocyte count was significantly lower in nonsurvivors than in survivors (4.2\%, IQR 2.4-5.85 versus 10.1\%, IQR5.65-23.4; $\mathrm{P}<0.05)$, which supports the theory that cellular immunity plays an important role in anti-pneumocystic therapy, particularly that involving $\mathrm{CD}^{+}{ }^{+} \mathrm{T}$ cells. Similarly, the $\mathrm{CD} 4^{+}$ $\mathrm{T}$ cell count in nonsurvivors was lower than that in survivors, although the difference was not significant statistically due to missing data. In univariate analysis, it was found that the neutrophil count in the BALF from nonsurvivors was significantly higher than that from survivors $(49.78 \pm 27.67$ versus $21.33 \pm 15.03 \%$; $\mathrm{P}<0.05)$, indicating the possibility that the elevation of the neutrophil count in BALF may be associated with a poor prognosis. The limited number of samples hampered the multivariate analysis.

In this study, 16 out of 18 patients were treated with co-trimoxazole as a first-line therapy; only five patients were treated with the intravenous form. According to the literature, the bioavailability via oral or intravenous administration is thought to be equivalent (30). Caspofungin, an inhibitor of fungal 1,3- $\beta$-D-glucan synthesis $(31,32)$, is effective for the treatment of invasive candidiasis and aspergillosis (33). There are some case reports indicating its effectiveness as a salvage or additional treatment for PCP $(34,35)$. Caspofungin has strong activity on cyst forms and weak activity on trophic forms, whereas TMP-SMZ mainly interferes with trophic forms. Theoretically, the concomitant use of TMP-SMZ and caspofungin, by fully inhibiting the organism life cycle, may provide synergistic activity against Pneumocysitis (36). In the present study, eight patients $(50 \%)$ received caspofungin therapy and $75 \%$ of them failed to survive. However, caspofungin is usually recommended in severe cases, and this may have affected the mortality rate. In addition, the number of patients was also limited in the present study. Adjunctive corticosteroids, in addition to antibiotics, are of substantial benefit in HIV-infected patients with moderate to severe hypoxemia $(37,38)$. In HIV-negative patients with PCP, there is no evidence that adjunctive steroid therapy is beneficial $(39,40)$. A review of 31 non-HIV-positive patients with confirmed PCP and hypoxemia found that those who received a higher dose of steroids had a shorter duration of mechanical ventilation and oxygen use (39). However, another similar study was unable to show an improvement in survival (40). A further study (42), which concluded that high-dose steroid therapy was associated with increased mortality in HIV-negative patients with PCP via a mechanism independent from an increased risk of infection, also supported this view. All of the patients analyzed in the present study were HIV-negative, and in some of them, PCP was associated with corticosteroid use; $72.22 \%$ of the patients received adjunctive corticosteroid therapy. Among them, nine (69.23\%) patients eventually experienced treatment failure, which might also be associated with their disease severity. Currently, whether to use corticosteroids or not and their appropriate doses requires serious consideration in severe cases of PCP in HIV-negative patients (41).

No common DHPS gene mutations of $P$. jirovecii were detected in the HIV-negative PCP patients in the present study. However, other mutations were present, the significance of which remains to be further identified. The elevation of neutrophil counts in BALF and reduction of lymphocyte counts in peripheral blood may be associated with poor outcome. The efficacy of adjunctive steroid therapy in HIV-negative PCP patients requires further investigation.

\section{References}

1. Krajicek BJ, Limper AH and Thomas CF Jr: Advances in the biology, pathogenesis and identification of Pneumocystis pneumonia. Curr Opin Pulm Med 14: 228-234, 2008.

2. Lane BR, Ast JC, Hossler PA, et al: Dihydropteroate synthase polymorphisms in Pneumocystis carinii. J Infect Dis 175: 482-485, 1997. 
3. Kazanjian P, Locke AB, Hossler PA, et al: Pneumocystis carinit mutations associated with sulfa and sulfone prophylaxis failures in AIDS patients. AIDS 12: 873-878, 1998.

4. Riebold D, Fritzsche C, Lademann M, Bier A and Reisinger EC: Pneumocystis jiroveci dihydropteroate synthase gene mutations at codon 171 but not at codons 55 or 57 detected in Germany. Clin Infect Dis 42: 582-583, 2006.

5. Helweg-Larsen J, Benfield TL, Eugen-Olsen J, Lundgren JD and Lundgren B: Effects of mutations in Pneumocystis carini dihydropteroate synthase gene on outcome of AIDS-associated P. carinii pneumonia. Lancet 354: 1347-1351, 1999.

6. Kazanjian PH, Fisk D, Armstrong W, et al: Increase in prevalence of Pneumocystis carinii mutations in patients with AIDS and P. carinii pneumonia, in the United States and China. J Infect Dis 189: 1684-1687, 2004.

7. Li K, He A, Cai WP, et al: Absence of Pneumocystis jirovecii dihydropteroate synthase gene mutations among samples from a group of AIDS patients in China. Scand J Infect Dis 41: 152-154, 2009.

8. Montes-Cano MA, de la Horra C, Martin-Juan J, et al: Pneumocystis jiroveci genotypes in the Spanish population. Clin Infect Dis 39: 123-128, 2004.

9. Huang L, Welsh DA, Miller RF, et al: Pneumocystis jirovecii dihydropteroate synthase gene mutations and human immunodeficiency virus-associated Pneumocystis pneumonia. J Eukaryot Microbiol 53 (Suppl 1): S114-S116, 2006.

10. Tyagi AK, Mirdha BR, Luthra K, et al: Dihydropteroate synthase (DHPS) gene mutation study in HIV-infected Indian patients with Pneumocystis jirovecii pneumonia. J Infect Dev Ctries 4: 761-766, 2010

11. Dini L, du Plessis M, Frean J and Fernandez V: High prevalence of dihydropteroate synthase mutations in Pneumocystis jirovecii isolated from patients with Pneumocystis pneumonia in South Africa. J Clin Microbiol 48: 2016-2021, 2010.

12. Brown PD, Freeman A and Foxman B: Prevalence and predictors of trimethoprim-sulfamethoxazole resistance among uropathogenic Escherichia coli isolates in Michigan. Clin Infect Dis 34: 1061-1066, 2002.

13. Walker DJ and Meshnick SR: Drug resistance in Pneumocystis carinii: an emerging problem. Drug Resist Updat 1: 201-204, 1998.

14. Edman JC, Edman U, Cao M, Lundgren B, Kovacs JA and Santi DV: Isolation and expression of the Pneumocystis carinii dihydrofolate reductase gene. Proc Natl Acad Sci USA 86: 8625-8629, 1989

15. Lane B, Hossler P, Bartlett M, et al: Sulfa resistance in mouse-derived Pneumocystis carinii. J Eukaryot Microbiol 43 : 39S, 1996.

16. Huang L, Crothers K, Atzori C, et al: Dihydropteroate synthase gene mutations in Pneumocystis and sulfa resistance. Emerg Infect Dis 10: 1721-1728, 2004

17. Beard CB, Carter JL, Keely SP, et al: Genetic variation in Pneumocystis carinii isolates from different geographic regions: implications for transmission. Emerg Infect Dis 6: 265-272, 2000.

18. Kazanjian P, Armstrong W, Hossler PA, et al: Pneumocystis carinii mutations are associated with duration of sulfa or sulfone prophylaxis exposure in AIDS patients. J Infect Dis 182: 551-557, 2000.

19. Takahashi T, Hosoya N, Endo T, et al: Relationship between mutations in dihydropteroate synthase of Pneumocystis carini f. sp. hominis isolates in Japan and resistance to sulfonamide therapy. J Clin Microbiol 38: 3161-3164, 2000.

20. van Hal SJ, Gilgado F, Doyle T, et al: Clinical significance and phylogenetic relationship of novel Australian Pneumocystis jirovecii genotypes. J Clin Microbiol 47: 1818-1823, 2009.

21. Thomas CF, Jr. and Limper AH: Current insights into the biology and pathogenesis of Pneumocystis pneumonia. Nat Rev Microbiol 5: 298-308, 2007.

22. de Boer MG, Gelinck LB, van Zelst BD, et al: $\beta$-D-glucan and $\mathrm{S}$-adenosylmethionine serum levels for the diagnosis of Pneumocystis pneumonia in HIV-negative patients: a prospective study. J Infect 62: 93-100, 2011.

23. Vogel MN, Weissgerber P, Goeppert B, et al: Accuracy of serum LDH elevation for the diagnosis of Pneumocystis jiroveci pneumonia. Swiss Med Wkly 141: w13184, 2011.
24. Held J, Koch MS, Reischl U, Danner T and Serr A: Serum $(1 \rightarrow 3)-\beta$-D-glucan measurement as an early indicator of Pneumocystis jirovecii pneumonia and evaluation of its prognostic value. Clin Microbiol Infect 17: 595-602, 2011.

25. Limper AH, Offord KP, Smith TF and Martin WJ II: Pneumocystis carinii pneumonia. Differences in lung parasite number and inflammation in patients with and without AIDS. Am Rev Respir Dis 140: 1204-1209, 1989.

26. Azoulay E, Bergeron A, Chevret S, Bele N, Schlemmer B and Menotti J: Polymerase chain reaction for diagnosing Pneumocystis pneumonia in non-HIV immunocompromised patients with pulmonary infiltrates. Chest 135: 655-661, 2009.

27. Wang JC, Huang MJ, A YJ, et al: Screening Pneumocystis carinii pneumonia in non-HIV-infected immunocompromised patients using polymerase chain reaction. Diagn Microbiol Infect Dis 64: 396-401, 2009.

28. Maskell NA, Waine DJ, Lindley A, et al: Asymptomatic carriage of Pneumocystis jiroveci in subjects undergoing bronchoscopy: a prospective study. Thorax 58: 594-597, 2003.

29. Sing A, Roggenkamp A, Autenrieth IB and Heesemann J: Pneumocystis carinii carriage in immunocompetent patients with primary pulmonary disorders as detected by single or nested PCR. J Clin Microbiol 37: 3409-3410, 1999

30. Kaplan JE, Benson C, Holmes KH, Brooks JT, Pau A and Masur H: Guidelines for prevention and treatment of opportunistic infections in HIV-infected adults and adolescents: recommendations from CDC, the National Institutes of Health, and the HIV Medicine Association of the Infectious Diseases Society of America. MMWR Recomm Rep 58: 1-207; quiz CE1-4, 2009.

31. Ripeau JS, Aumont F, Belhumeur P, Ostrosky-Zeichner L, Rex JH and de Repentigny L: Effect of the echinocandin caspofungin on expression of Candida albicans secretory aspartyl proteinases and phospholipase in vitro. Antimicrob Agents Chemother 46: 3096-3100, 2002.

32. Deresinski SC and Stevens DA: Caspofungin. Clin Infect Dis 36: 1445-1457, 2003

33. Espinel-Ingroff A: Comparison of in vitro activities of the new triazole SCH56592 and the echinocandins MK-0991 (L-743,872) and LY303366 against opportunistic filamentous and dimorphic fungi and yeasts. J Clin Microbiol 36: 2950-2956, 1998

34. Mu XD, Que CL, He B, Wang GF and Li HC: Caspofungin in salvage treatment of severe Pneumocystis pneumonia: case report and literature review. Chin Med J (Engl) 122: 996-999, 2009.

35. Zhang JC, Dai JY, Fan J and Wu XP: The treatment of Pneumocystis carinii pneumonia with caspofungin in elderly patients: a case report and literature review. Zhonghua Jie $\mathrm{He} \mathrm{He}$ Hu Xi Za Zhi 29: 463-465, 2006 (In Chinese).

36. Powles MA, Liberator P, Anderson J, et al: Efficacy of MK-991 (L-743,872), a semisynthetic pneumocandin, in murine models of Pneumocystis carinii. Antimicrob Agents Chemother 42: 1985-1989, 1998

37. Calderón EJ, Gutiérrez-Rivero S, Durand-Joly I and Dei-Cas E: Pneumocystis infection in humans: diagnosis and treatment. Expert Rev Anti Infect Ther 8: 683-701, 2010.

38. Briel M, Boscacci R, Furrer H and Bucher HC: Adjunctive corticosteroids for Pneumocystis jiroveci pneumonia in patients with HIV infection: a meta-analysis of randomised controlled trials. BMC Infect Dis 5: 101, 2005.

39. Pareja JG, Garland R and Koziel H: Use of adjunctive corticosteroids in severe adult non-HIV Pneumocystis carinii pneumonia. Chest 113: 1215-1224, 1998.

40. Delclaux C, Zahar JR, Amraoui G, et al: Corticosteroids as adjunctive therapy for severe Pneumocystis carinii pneumonia in non-human immunodeficiency virus-infected patients: retrospective study of 31 patients. Clin Infect Dis 29: 670-672, 1999.

41. Carmona EM and Limper AH: Update on the diagnosis and treatment of Pneumocystis pneumonia. Ther Adv Respir Dis 5: 41-59, 2011

42. Lemiale V, Debrmetz A, Delannoy A, Alberti C and Azoulay E: Adjunctive steroid in HIV-negative patients with severe Pneumocystis pneumonia, Respir Res 14: 87, 2013. 